\title{
Deterioration of Visuospatial Associative Memory Following a First Psychotic Episode: A Long-term Follow-up Study
}

Cassandra M J Wannan ${ }^{1}$, Cali F Bartholomeusz ${ }^{1,2,3}$, Vanessa L Cropley ${ }^{1}$, Tamsyn E Van

Rheenen $^{1,7,8}$, Anita Panayiotou ${ }^{1}$, Warrick J. Brewer ${ }^{2}$, Tina M. Proffitt ${ }^{2}$, Lisa Henry ${ }^{2,3}$, Meredith

G Harris ${ }^{4}$, Dennis Velakoulis ${ }^{1}$, Patrick McGorry ${ }^{2,3}$, Christos Pantelis ${ }^{1,5 *}$, Stephen J Wood ${ }^{1,2,3,6 *}$

* joint senior authors

${ }^{1}$ Melbourne Neuropsychiatry Centre, Department of Psychiatry, The University of

Melbourne \& Melbourne Health, Carlton South, Vic, Australia

${ }^{2}$ Orygen, The National Centre of Excellence in Youth Mental Health, Parkville, Victoria,

Australia

${ }^{3}$ The Centre for Youth Mental Health, The University of Melbourne, Parkville, Victoria,

Australia

${ }^{4}$ School of Public Health, University of Queensland \& QCMHR, Archerfield, Queensland,

Australia

${ }^{5}$ Centre for Neural Engineering, Department of Electrical and Electronic Engineering,

University of Melbourne, South Carlton, Victoria, Australia

${ }^{6}$ School of Psychology, University of Birmingham, Edgbaston, UK

${ }^{7}$ Centre for Mental Health, Faculty of Health, Arts and Design, School of Health Sciences,

Swinburne University, Melbourne, Australia

${ }^{8}$ Cognitive Neuropsychiatry Laboratory, Monash Alfred Psychiatry Research Centre (MAPrc),

The Alfred Hospital and Central Clinical School, Monash University, Melbourne, Australia.

Correspondence: Cassandra Wannan, Melbourne Neuropsychiatry Centre, Department of Psychiatry, The University of Melbourne \& Melbourne Health, Carlton South, Vic, Australia, 3053 (cwannan@student.unimelb.edu.au)

Word count: 3880 
Background. Cognitive deficits are a core feature of schizophrenia, and impairments in most domains are thought to be stable over the course of the illness. However, cross-sectional evidence indicates that some areas of cognition, such as visuospatial associative memory, may be preserved in the early stages of psychosis, but become impaired in later established illness stages. This longitudinal study investigated change in visuospatial and verbal associative memory following psychosis onset.

Methods. 95 first-episode psychosis (FEP) patients and 63 healthy controls (HC) were assessed on neuropsychological tests at baseline, with 38 FEP and $22 \mathrm{HCs}$ returning for follow-up assessment at 5-11 years. Visuospatial associative memory was assessed using the Cambridge Neuropsychological Test Automated Battery (CANTAB) Visuospatial Paired-Associate Learning (PAL) task, and verbal associative memory was assessed using Verbal Paired Associates (VPA) subtest of the Wechsler Memory Scale - Revised.

Results. Visuospatial and verbal associative memory at baseline did not differ significantly between FEP patients and healthy controls. However, over follow-up, visuospatial associative memory deteriorated significantly for the FEP group, relative to healthy individuals. Conversely, verbal associative memory improved to a similar degree observed in healthy controls. In the FEP cohort, visuospatial (but not verbal) associative memory ability at baseline was associated with functional outcome at follow-up.

Conclusions. Areas of cognition that develop prior to psychosis onset, such as visuospatial and verbal associative memory, may be preserved early in the illness. Later deterioration in visuospatial memory ability may relate to progressive structural and functional brain abnormalities that occur following psychosis onset. 
Introduction

Cognitive deficits are considered to be a core feature of the schizophrenia-spectrum, with impairments apparent in both first-episode psychosis (FEP) patients, and in individuals with established illness (Aas et al., 2014; Bora et al., 2009). Although deficits are observed across virtually all cognitive domains, the greatest levels of impairment are seen in attention, executive function, verbal fluency, and verbal learning and memory (Fatouros-Bergman et al., 2014; Bora et al., 2009; Aas et al., 2014).

Currently, deficits in cognition in schizophrenia are thought to be present at the time of illness onset and to remain stable over its course (Bora \& Murray, 2013). This is consistent with the prevailing neurodevelopmental hypothesis, which posits that abnormal brain development leads to arrested maturation of cognitive abilities in individuals who go on to develop schizophrenia (Weinberger, 1987)'(Murray \& Lewis, 1987). Indeed, the evidence from longitudinal studies shows that the cognitive performance of individuals at ultra-high risk (UHR) of developing psychosis and FEP patients improves over time (Bora \& Murray, 2013). These findings indicate that cognitive deficits are present prior to the onset of prodromal symptoms, with no evidence for a decline in functioning as the illness progresses.

Cross-sectional studies on the other hand, provide evidence for cognitive deterioration following psychosis onset (Napal et al., 2012), at least in some areas of cognitive functioning. These studies, which typically compare cognitive functioning in early illness stages with that of chronic schizophrenia patients, tend to report more severe cognitive impairment in individuals with chronic or multi-episode schizophrenia (Keefe, 2014; Napal et al., 2012; Braw et al., 2008). A recent review of cognitive ability at different illness stages revealed that individuals at UHR of developing psychosis demonstrated only mild cognitive impairments, with performance approximately 0.5 standard deviations below that of healthy individuals 
(Keefe, 2014). Recent onset patients, on the other hand, demonstrated cognitive performance approximately 1.5 standard deviations below that of healthy comparison subjects. However, the largest deficits were observed in individuals with chronic schizophrenia, whose performance sat around 2.5 standard deviations below that of healthy individuals. Taken together, these findings suggest that cognition may deteriorate as the severity of illness increases, or that poor clinical outcome is associated with poor cognitive performance (Bodnar et al., 2008; Carlsson et al., 2006; Leeson et al., 2009a).

Visuospatial associative memory is one area of cognition that has been found to be more impaired in individuals with established schizophrenia than in FEP patients (Wood et al., 2002; Barnett et al., 2005; Stip et al., 2005; Donohoe et al., 2008). A previous cross-sectional study found that performance on the Cambridge Neuropsychological Test Automated Battery (CANTAB) paired-associates learning (PAL) task was preserved in FEP patients, who performed at a level similar to that of healthy controls (Wood et al., 2002). Conversely, chronic patients demonstrated impaired PAL performance when compared to both healthy controls and to FEP patients. This discrepancy in cognitive performance between cohorts could indicate a deteriorating course - however, it could also be that poorer PAL performance in FEP is predictive of chronic illness outcome. To date, PAL performance has not been measured longitudinally over time in first-episode schizophrenia patients. Therefore, this question of a 'deteriorating' visuospatial memory course versus the prognostic value of low PAL ability remains to be directly assessed.

Here we report the results of the first longitudinal study of visuospatial associative memory performance in FEP patients, followed up over five to ten years. Our aim was to investigate the longitudinal course of visuospatial associative memory ability following psychosis onset, and to compare this with the course of verbal associative memory ability over the same 
period. Based on previous cross-sectional findings, we hypothesised that visuospatial associative memory would deteriorate over the follow-up period for the FEP patients, whereas the performance of the healthy controls would remain stable. Verbal associative memory was expected to remain stable, or to improve, in both FEP patients and healthy controls over the follow-up period.

\section{Method}

\section{Participants and Clinical Assessment}

Table 1 presents the demographic and clinical characteristics of the sample. At baseline, 95 individuals experiencing a first episode psychosis (FEP) were recruited from Orygen Youth Health's Early Psychosis Prevention and Intervention Centre (EIC), Melbourne, Australia between 1993-2001. Diagnoses included schizophrenia $(N=19)$, schizophreniform disorder $(N=45)$, schizoaffective disorder $(N=17)$, delusional disorder $(N=2)$, brief psychotic disorder $(N=4)$, psychotic disorder not otherwise specified $(N=6)$, and substance-induced psychosis $(N=2)$. FEP-specific inclusion criteria were; (1) current DSM-III (American Psychiatric Association, 1987) Axis I diagnosis of a psychotic disorder; and (2) less than 6 months of neuroleptic treatment. The current study also excluded FEP patients with a primary mood diagnosis (bipolar disorder and depression). The Royal Park Multidiagnostic Instrument for Psychosis (RPMIP)(Mcgorry et al., 1990) and the Structured Diagnostic Interview for DSM-III (SCID-I/P)(First et al., 2002) were used to determine diagnosis. Further information about the methodology has been described previously (Henry et al., 2007). Healthy control $(\mathrm{HC})$ participants $(N=63)$ were recruited from the same catchment area as patients. HCs with a history of psychiatric illness were excluded. Exclusion criteria for FEP and $\mathrm{HC}$ included: significant head injury and/or loss of consciousness for $>1$ minute; preferred language other than English; known history of learning disability; estimated premorbid IQ $<70$ based on the National Adult Reading Test (NART)(Nelson \& Willison, 
1991); DSM diagnosis of substance abuse or dependence; neurological disease; baseline age $>45$. All participants provided informed consent and had adequate English comprehension.

Follow-up assessments were carried out between 1998 and 2005. Thirty-eight FEP and 22 HCs completed follow-up cognitive assessment, although a number of participants did not complete all cognitive tasks (see Table 2). For the FEP patients, level of functioning at followup was measured using the Social and Occupational Functioning Assessment Scale (SOFAS) (Goldman et al., 1992).

\section{Cognitive measures}

Estimated premorbid IQ was measured with the NART(Nelson \& Willison, 1991). NART scores were converted to Wechsler Adult Intelligence Scale-Revised intelligence quotient (WAIS-R IQ)(Wechsler, 1981) scores. Four subtests of the WAIS-R were used to obtain an estimate of current full scale IQ.

Visuospatial associative memory was assessed using the Visuospatial Paired-Associate Learning (PAL) Task from the CANTAB (Cambridge Cognition, 2016). This task uses cued recall of a series of patterns at different spatial locations. In the early stages of the task, six white boxes are spaced evenly on the screen, and opened up in a random order to reveal a coloured pattern inside. In the first trial, only one box contains a coloured pattern. Once all of the boxes have opened, the pattern is displayed in the centre of the screen and the participant is required to touch the box in which it was located. The number of patterns increases in subsequent trials, and the final stage of the task requires participants to remember the locations of 8 different patterns. The number of errors on the final stage of the task ( 8 shapes) was the variable of interest for the current study. Participants who did not reach the 8 shape stage of the task were given an adjusted score of 70 as described in 
the CANTAB administration guide (Cambridge Cognition, 2012). This adjustment is calculated using the following formula:

$\left(\right.$ number of patterns not attempted (8) $\left.-\frac{\text { number of boxes (8) }}{\text { number of patterns (8) }}\right) \times \quad$ total number of possible trials (10)

Basic visual recognition was measured with the Pattern Recognition (PR) task from the CANTAB (Cambridge Cognition 2016). In a white box in the centre of the screen, a series of 12 patterns were displayed one by one. Patterns were designed so that they cannot be easily verbalised. In the recognition phase, the participant is required to choose between a novel pattern and a pattern that they have already seen. Patterns are presented in reverse order in the recognition phase. This sub-test is then repeated with a second set of patterns, and participants are given a score out of 24 .

Verbal associative memory was assessed using the semantically-unrelated (difficult) pairs from the from the Wechsler Memory Scale-Revised (WMS-R) Verbal Paired Associates (VPA) task (Wechsler, 1987). In this task, participants were first read a list of 4 word pairs. The examiner then read the first word of each pair, and the participant is asked to provide the corresponding word. Three trials were completed.

\section{Data Analysis}

Group differences between FEP patients and healthy controls on demographic measures were examined using chi-square tests for categorical data, and independent groups t-tests for continuous variables.

Due to the fact that PAL errors are recorded as a count variable, and data were overdispersed (the variance was substantially greater than the mean), generalised estimating equations (GEE) negative binomial regression was determined to be the most 
appropriate method for examining change in PAL scores over time. Stepwise backward selection criteria were employed to determine the final model: all independent variables (IV) were initially entered into the model and then variables with a $p$ value $>0.05$ were removed. Group and time (time 1, time 2), as well as the group time interaction, were included as IVs in each model. Initial covariates included age, IQ, years of education, sex, length of followup, and PR scores. PR was included as a covariate in the analysis of PAL performance as it has been found to predict performance on the PAL task in previous studies (Wood et al.,2002). GEE allows for missing data at each time-point, however data must be missing completely at random. As data-screening analyses confirmed that missing data at follow-up were not related to any variables of interest (including: age, sex, diagnosis, baseline cognition scores [PAL errors, verbal memory scores, IQ, estimated premorbid IQ], or years of education), data were deemed missing completely at random, and participants with missing data were thus included in the analysis.

A generalised linear mixed model was used to analyse change in VPA performance over time. This model allows the slope or intercept to vary across participants as a random effect (Laird \& Ware, 1982). Again, stepwise backward selection criteria were employed to determine the final model. In the initial model, VPA scores were included as the dependent variable, and group, time (time 1 , time 2 ), group $x$ time, age, $I Q$, and years of education were included as fixed effects. Our analyses included all available data collected for each participant at each time-point.

Relationships between neuropsychological test scores (PAL and VPA) and social and occupational functioning at follow-up were analysed using Spearman's rank order correlations. 


\section{Results}

Demographics

FEP patients had a significantly shorter follow-up interval than HCs, and a significantly lower age at follow-up (see Table 1). HCs also completed more years of education, and had higher estimated premorbid IQ scores than FEP patients. Neuropsychological assessment scores at baseline and follow-up are displayed in Table 2.

\section{Visuospatial associative memory}

Table 3 displays the results of the generalised estimating equation model for PAL

performance. IQ, sex, and PR performance were included as covariates in the final model. IQ and PR scores were negatively correlated with PAL performance, with lower scores associated with more errors on the PAL task. There was also a main effect of sex, with males making more errors than females. A significant group $\mathrm{p}_{\mathrm{x}}$ time interaction was also observed (Wald $\left.\chi^{2}(1)=6.02, p=.014\right)$ : At baseline, there was no difference between FEP individuals and HCs in terms of PAL errors, however the HC group demonstrated slight improvement on the PAL task over time, whereas performance of the FEP group deteriorated from baseline to follow-up (see Figure 1A).

\section{Verbal Associative Memory}

Table 4 displays the generalised linear mixed model for VPA performance. IQ was included as a covariate in the final model as IQ scores were positively related to VPA scores. A main effect of time was also observed, with both groups' performance improving over the follow-

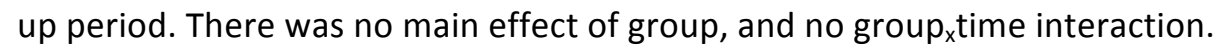

\section{Correlations with Functioning}

For patients, number of errors on the PAL task at baseline was significantly negatively 
correlated with level of functioning at follow-up, $r_{s}(26)=-0.38 p=.045$. There was no correlation between baseline VPA performance and SOFAS scores at follow-up. Number of PAL errors at follow-up was significantly negatively correlated with SOFAS scores at followup, $r_{s}(34)=-0.37 p=.027$. VPA scores at follow-up were also significantly correlated with SOFAS scores at follow-up, $r_{s}(35)=-0.41 p=.012$

\section{Discussion}

In this longitudinal study of individuals with FEP disorders, we examined changes in visuospatial and verbal associative memory abilities over a follow-up period of 5 to 11 years. Our findings revealed no difference in either visuospatial or verbal associative memory scores in FEP patients relative to HCs at baseline. However, we identified a deteriorating trajectory in visuospatial associative memory compared with healthy controls subjects. In contrast change in verbal associative memory did not differ from healthy control subjects, with both groups showing improved ability over time.

While previous studies have typically found some aspects of memory to be impaired at illness onset (Bora \& Murray, 2013; Aas et al.,2014), the results of the current study, and of other similar studies of associative memory (Bartholomeusz et al., 2011; Brewer et al., 2005; Williams, Avery, Woolard, \& Heckers, 2012; Wood et al., 2002, 2007), indicate that both visual and verbal associative memory are preserved in the early stages of psychosis. Chronic schizophrenia patients, on the other hand, have been found to demonstrate impairments in both visual (Armstrong, Williams, \& Heckers, 2012; Armstrong, Kose, Williams, Woolard, \& Heckers, 2012; Donohoe et al., 2008; Toulopoulou, Rabe-Hesketh, King, Murray, \& Morris, 2003; Wood et al., 2002) and verbal associative memory (Elvevag et al.,2000; Toulopoulou et al.,2003), which suggests that associative memory ability may deteriorate over the course of psychotic illness. 
Consistent with previous cross-sectional findings (Wood et al., 2002; Barnett et al., 2005;

Stip et al., 2005; Donohoe et al., 2008), the current study found that visuospatial associative memory ability was preserved at baseline in the FEP cohort. In this first longitudinal study of PAL performance from psychosis onset, visuospatial associative memory ability deteriorated significantly over a 5-11 year follow-up period in patients having a schizophrenia spectrum disorder at baseline. This decline occurred despite stability in pattern recognition performance over the same period in both FEP and healthy controls, indicating that the decline in visuospatial associative memory performance is not accompanied by any changes in basic visual recognition. Deterioration in visuospatial associative memory challenges the 'dominant' notion of stable cognitive deficits in schizophrenia (Bora \& Murray 2013), and highlights that different areas of cognition may have different trajectories over the course of psychotic illness. That is, while some cognitive domains are impaired at the earliest stages of the illness, other areas of cognition are preserved at illness onset and deteriorate only as the illness progresses (Pantelis, Wannan, Bartholomeusz, Allott, \& McGorry, 2015). In order to understand these differing trajectories, it is important to consider individual cognitive tasks within the context of normal development, while also taking into account the brain changes that have been found to occur in the period following illness onset (Pantelis et al., 2005; Pantelis, Yucel, et al., 2009; Pantelis, Wannan, Bartholomeusz, Allott, \& McGorry, 2015).

We have previously argued that there is an interaction between the age of illness onset and stage of brain development, which may result in a relatively greater impact on brain structures and functions that are maturing around the time of illness onset (Gogtay, Vyas, Testa, Wood, \& Pantelis, 2011; Pantelis et al., 2005; Pantelis, Yucel, et al., 2009; Pantelis et al., 2015). Thus, areas of cognition that are continuing to mature are likely to have their development interrupted by illness onset (possibly before overt symptoms), whereas areas 
of cognition that develop early, prior to illness onset, may be preserved. Spatial working memory ability, for example, which typically does not fully mature until the mid-20s (De Luca et al.,2003), and may therefore be interrupted by psychosis onset, has been found to be impaired to a similar extent in FEP patients and chronic schizophrenia patients (Pantelis, Wood, et al., 2009; Wood et al., 2002). Conversely, associative memory ability develops early in life, with performance increasing steeply between ages 8 and 11 and plateauing thereafter (Thaler et al., 2013). It is possible that this ability has finished maturing prior to illness onset, and is therefore unaffected in the early stages of the illness. This was also observed on a task of attentional set-shifting ability (Pantelis, Wood, et al., 2009), which also matures early (De Luca et al., 2003).

Previous studies have argued that cognitive impairments observed in first-episode patients may reflect underlying deficits in executive functions or information processing, which are related to prefrontal cortex dysfunction (Leeson et al., 2009b; Leeson et al., 2010). However, tasks that index medial temporal lobe functioning, such as verbal memory retention, appear to be independent of this relationship (Leeson et al., 2009b). Our results suggests that the deficits observed in PAL are likewise independent of such underlying deficits in executive functioning, and this may be due to the heavy reliance on medial temporal lobe structures required for this task. The brain network that is implicated in performance on the PAL task is the hippocampal-prefrontal pathway (De Rover et al., 2011; Barnett et al., 2016) which has been shown to be disrupted in individuals with schizophrenia (Sigurdsson \& Duvarci, 2015). Activity in this pathway is highly sensitive to stress (Godsil et al., 2013), and it is therefore possible that the acute stress experienced at the onset of psychosis may lead to increasingly aberrant functional coupling between the hippocampus and prefrontal cortex and, consequently, deteriorating visuospatial associative memory ability. Regions of the temporal lobes, such as the parahippocampus and the entorhinal cortex, are also implicated in PAL 
performance (Owen, Sahakian, Semple, Polkey, \& Robbins 1995; De Rover et al., 2011), and have also been shown to be abnormal in schizophrenia (Joyal et al., 2002; Bartholomeusz et al., 2016). Longitudinal studies of brain structure in individuals with schizophrenia have identified progressive loss of grey matter in regions relevant to the PAL task, including in the prefrontal cortex and the medial temporal lobe (Bartholomeusz et al., 2016; Radua et al., 2012; Vita, De Peri, Deste, \& Sacchetti, 2012). Thus, while visuospatial associative memory ability is preserved at the time of illness onset, progressive deterioration, both structurally and functionally, in brain regions relevant to this area of cognition may impact on functioning as the illness progresses.

Further, we found that visuospatial associative memory performance at baseline and followup was associated with functional outcome at follow-up. A previous study of FEP patients by Barnett et al.,(2005) found that individuals who failed this same task scored higher on negative symptom and psychopathology measures, had poorer levels of global functioning, and higher disability scores. In chronic patients, visuospatial associative memory performance at baseline predicted improvement in daily living autonomy and social competence following participation in a rehabilitation program (Prouteau et al., 2005). The current study adds to these findings by demonstrating that visuospatial associative memory ability early in a psychotic illness can predict social and occupational functioning over long follow-up periods of 5-11 years. Thus, poor performance on this task at the time of psychosis onset may help identify individuals having poor long-term functional outcome. In contrast, baseline performance on the verbal paired-associates was not associated with later social and occupational functioning, suggesting that its use as a predictive tool is limited in psychosis populations.

The observed deterioration of visuospatial associative memory contrasts with the relatively 
preserved verbal associative memory performance observed over the same period in the FEP cohort. Thus, the observed improvement in verbal associative memory over time was in accord with improved ability in controls. While a number of longitudinal studies have observed similar improvement in verbal memory performance following psychosis onset (Rodríguez-Sánchez et al.,2013; Olivier et al., 2015), what differentiates the current findings from those of the majority of previous studies is that, after accounting for general intellectual impairment, the patient group did not demonstrate deficits on the verbal associative memory task at either baseline or follow-up. While this result is surprising, it is in line with an earlier cross-sectional study of verbal memory abilities in FEP patients and individuals with chronic schizophrenia (Wood et al., 2007), which found preserved verbal associative memory ability in both patient cohorts relative to healthy controls. Preservation of verbal associative memory may simply be related to the relative ease of the VPA task compared to the PAL task. That is, this task may not be sensitive enough to psychosis-related brain abnormalities to detect memory impairments above and beyond those that result from generalised intellectual impairment. The ease of the VPA task may, in-part, reflect the fact that the attentional load of this task is likely to be much lower than that of the CANTAB PAL task. As attention has been found to be highly impaired in individuals with schizophrenia (Aas et al., 2014; Braw et al., 2008; Fioravanti et al., 2012), tasks with a high attentional load are more likely to be impaired in these individuals. Previous studies that have identified impairments in VPA performance in FEP have tended not to include measures of IQ or attention as covariates in their analyses (e.g. Hutton et al., 1998; Goldberg et al., 1990), and it is therefore difficult to separate out memory impairments from impairments in general intellectual functioning.

The results of the current study should be considered in the context of several limitations. The small number of participants (particularly healthy individuals) completing the PAL task 
at follow-up may limit the generalisability of the findings. However, due to the long followup duration, a high attrition rate is not unexpected. Additionally, follow-up assessment was only conducted at one time-point, and it is therefore impossible to determine the timing and rate of the changes that were observed in cognitive performance at follow-up. Increasing the number of follow-up time-points would help identify when cognitive changes occur following psychosis onset, and the relative speed with which they occur, especially given brain changes in early illness stages are shown to occur in a relatively short timeframe (Bartholomeusz et al., 2016).

In summary, the current study has found that visuospatial associative memory was preserved at baseline in FEP patients, with significant deterioration occurring over the 5 - to 11-year follow-up period relative to healthy individuals. Baseline performance on the PAL task was also found to be a predictor of later social and occupational functioning. Verbal associative memory, on the other hand, was unimpaired at both time-points, and was found to improve over follow-up to a similar degree observed in healthy controls. Baseline verbal associative memory ability was not associated with functioning at follow-up. These findings highlight the importance of taking the normal maturational trajectories of different areas of cognition into account, with evidence suggesting that areas of cognition that reach maturity earlier in life are likely to be preserved at the time of illness onset, but may be impacted by factors relating to the illness or its treatment, leading to deterioration of these abilities over time. These findings may also have implications for the remediation strategies used to treat cognitive impairments in schizophrenia; while these strategies have, to date, largely focused on deficits, it is possible that different approaches are required for areas of cognition with different trajectories (Pantelis et al., 2015). Namely, preventing deterioration in areas of cognition that are relatively unaffected at illness onset, particularly those that are closely related to functional outcome, should be an important treatment goal. 
Acknowledgments

The study was supported by NHMRC Project Grants (IDs: 970598, 981112, 145627) and NHMRC Program Grants (ID: 350241, 566529). Prof C. Pantelis was supported by a NHMRC Senior Principal Research Fellowship (628386 \& 1105825), and a Brain and Behavior Research Foundation (NARSAD) Distinguished Investigator Award (US; Grant ID: 18722). Prof Patrick McGorry was supported by a NHMRC senior principal research fellowship. Dr Van Rheenen was supported by a National Health and Medical Research Council (NHMRC) Early Career Fellowship (APP1088785).

Conflicts of Interest: None 


\section{References}

Aas, M., Dazzan, P., Mondelli, V., Melle, I., Murray, R.M. \& Pariante, C.M. (2014). A systematic review of cognitive function in first-episode psychosis, including a discussion on childhood trauma, stress, and inflammation. Frontiers in Psychiatry, 4, 1-13.

American Psychiatric Association (1987). Diagnostic and Statistical Manual of Mental Disorders 3rd ed., Washington, DC: American Psychiatric Association.

Armstrong, K., Kose, S., Williams, L., Woolard, A. \& Heckers, S. (2012). Impaired associative inference in patients with schizophrenia. Schizophrenia Bulletin, 38(3), 622-629.

Armstrong, K., Williams, L.E. \& Heckers, S. (2012). Revised associative inference paradigm confirms relational memory impairment in schizophrenia. Neuropsychology, 26(4), 451-458.

Barnett, J.H., Blackwell, A.D., Sahakian, B.J. \& Robbins, T.W. (2016). The Paired Associates Learning (PAL) Test: 30 Years of CANTAB Translational Neuroscience from Laboratory to Bedside in Dementia Research. Current Topics in Behavioral Neuroscience, 28, 449474.

Barnett, J.H., Sahakian, B.J., Werners, U., Hill, K.E., Brazil, R., Gallagher, O., Bullmore, E.T. \& Jones, P.B. (2005). Visuospatial learning and executive function are independently impaired in first-episode psychosis. Psychological Medicine, 35, 1031-1041.

Bartholomeusz, C.F., Proffitt, T.M., Savage, G., Simpson, L., Markulev, C., Kerr, M., McConchie, M., McGorry, P.D., Pantelis, C., Berger, G.E. \& Wood, S.J. (2011). Relational memory in first episode psychosis: implications for progressive hippocampal dysfunction after illness onset. Australian and New Zealand Journal of Psychiatry, 45, 206-213.

Bartholomeusz, C.F., Cropley, V., Wannan, C., Di Biase, M., McGorry, P.D. \& Pantelis, C. (2016). Structural neuroimaging across early stage psychosis: Aberrations in neurobiological trajectories and implications for the staging model. Australian and New Zealand Journal of Psychiatry, October, .1-22.

Bodnar, M., Malla, A.K., Joober, R. \& Lepage, M. (2008). Cognitive markers of short-term clinical outcome in first-episode psychosis. The British Journal of Psychiatry, 193, 297304.

Bora, E. \& Murray, R.M. (2013). Meta-analysis of cognitive deficits in ultra-high risk to psychosis and first-episode psychosis: Do the cognitive deficits progress over, or after, the onset of psychosis? Schizophrenia Bulletin, 40(4), 744-755.

Bora, E., Yucel, M. \& Pantelis, C. (2009). Cognitive functioning in schizophrenia, schizoaffective disorder and affective psychoses: Meta-analytic study. The British Journal of Psychiatry, 195, 475-482.

Braw, Y., Bloch, Y., Mendelovich, S., Ratzoni, G., Gal, G., Harari, H., Tripto, A. \& Levkovitz, Y. (2008). Cognition in young schizophrenia outpatients: Comparison of first-episode with multiepisode patients. Schizophrenia Bulletin, 34(3), 544-554. 
Brewer, W.J., Francey, S.M., Wood, S.J., Jackson, H.J., Pantelis, C., Phillips, L.J., Yung, A.R., Anderson, V.A. \& McGorry, P.D. (2005). Memory impairments identified in people at ultra-high risk for psychosis who later develop first-episode psychosis. American Journal of Psychiatry, 162, 71-78.

Cambridge Cognition, (2016). CANTAB [Cognitive assessment software]. Available at: www.cantab.com [Accessed October 7, 2016].

Cambridge Cognition, (2012). CANTAB eclipse Test Administration Guide, Bottisham: Cambridge Cognition Limited.

Carlsson, R., Nyman, H., Ganse, G. \& Cullberg, J. (2006). Neuropsychological functions predict 1- and 3-year outcome in first-episode psychosis. Acta Psychiatrica Scandinavica, 113, 102-111.

de Rover, M., Pironti, V.A., McCabe, J.A., Acosta-Caberonero, J., Arana, F.S., Morein-Zamir, S., Hodges, J.R., Robbins, T.W., Fletcher, P.C., Nestor, P.J., \& Shahakian, B.J. (2011). Hippocampal dysfunction in patients with mild cognitive impairment: a functional neuroimaging study of a visuospatial paired associates learning task. Neuropsychologica, 49:7, 2060-2070.

Donohoe, G., Spoletini, I., McGlade, N., Behan, C., Hayden, J., O'Donoghue, T., Peel, R., Haq, F., Walker, C., O'Callaghan, E., Spalletta, G., Gill, M. \& Corvin, A. (2008). Are relational style and neuropsychological performance predictors of social attributions in chronic schizophrenia? Psychiatry research, 161, 19-27.

Elvevag, B., Egan, M.F. \& Goldberg, T.E. (2000). Paired-associate learning and memory interference in schizophrenia. Neuropsychologia, 38, 1565-1575.

Fatouros-Bergman, H., Cervenka, S., Flyckt, L., Edman, G. \& Farde, L. (2014). Meta-analysis of cognitive performance in drug-naive patients with schizophrenia. Schizophrenia Research, 158, 156-162.

Fioravanti, M., Bianchi, V. \& Cinti, M.E. (2012). Cognitive deficits in schizophrenia: an updated metanalysis of the scientific evidence. BMC psychiatry, 12, 64-83.

First, M. et al., 2002. Structured Clinical Interview for DSM-IV-TR Axis I Disorders, Research Version, Patient Edition, New York, NY: New York State Psychiatric Institute.

Godsil, B.P., Kiss, J.P., Spedding, M. \& Jay, T.M. (2013). The hippocampal-prefrontal pathway: The weak link in psychiatric disorders? European Neuropsychopharmacology, 23, 1165-1181.

Gogtay, N., Vyas, N.S., Testa, R., Wood, S.J. \& Pantelis, C. (2011). Age of onset of schizophrenia: perspectives from structural neuroimaging studies. Schizophrenia Bulletin, 37(3), 504-513.

Goldberg, T.E., Ragland, J.D., Torrey, E.F., Gold J.M., Bigelow, L.B., \& Weinberger, D.R. (1990). Neuropsychological assessment of monozygotic twins discordant for schizophrenia. Archives of General Psychiatry, 47:11, 1066-1072. 
Goldman, H.H., Skodol, A. \& Lave, T.R. (1992). Revising axis V for DSM-IV: A review of measures of social functioning. American Journal of Psychiatry, 149, 1148-1156.

Henry, L.P., Harris, M.G., Amminger, G.P., Yuen, H.P., Harrigan, S.M., Lambert, M., Conus, P., Schwartz, O., Prosser, A., Farrelly, S., Purcell, R., Herrman, H., Jackson, H.J. \& McGorry, P.D. (2007). Early Psychosis Prevention and Intervention Centre long-term follow-up study of first-episode psychosis: methodology and baseline characteristics. Early Intervention in Psychiatry, 1, 49-60.

Hutton, S. B., Puri, B. K., Duncan, L. J., Robbins, T. W., Barnes, T. R., \& Joyce, E. M. (1998). Executive function in first-episode schizophrenia. Psychological Medicine, 28, 463-473.

Joyal, C.C., Laakso, M.P., Tiihonen, J., Syvalahti, E., Vilkman, H., Laakso, A., Alakare, B., Rakkolainen, V., Salokangas, R.K.R., Hietala, J. (2002). A volumetric MRI study of the entorhinal cortex in first-episode neuroleptic-naïve schizophrenia. Society of Biological Psychiatry, 51:12, 1005-1007.

Keefe, R.S.E. (2014). The longitudinal course of cognitive impairment in schizophrenia: An examination of data from premorbid through posttreatment phases of illness. Journal of Clinical Psychiatry, 75(SUL. 2), 8-13.

Laird, N.M. \& Ware, J.H. (1982). Random-Effects Models for Longitudinal Data. Biometrics, $38,963-974$.

Leeson, V.C., Barnes, T.R.E., Hutton, S.B., Ron, M.A. \& Joyce, E.M. (2009a). IQ as a predictor of functional outcome in schizophrenia: A longitudinal, four-year study of first-episode psychosis. Schizophrenia Research, 107, 55-60.

Leeson, V.C., Robbins, T.W., Franklin, C., Harrison, M., Harrison, I., Ron, M.A., Barnes, T.R.E., \& Joyce, E.M. (2009b). Dissociation of long-term verbal memory and frontoexecutive impairment in first-episode psychosis. Psychological Medicine, 39:11, 17991808.

Leeson, V.C., Barnes, T.R.E., Harrison, M., Matheson, E., Harrison, I., Mutsatsa, S. H., Ron, M.A., \& Joyce, E. M. (2010). The relationship between IQ, memory, executive function, and processing speed in recent-onset psychosis: 1-year stability and clinical outcome. Schizophrenia Bulletin, 36:2, 400-409.

de Luca, C.R., Wood, S.J., Anderson, V., Buchanan, J.A., Proffitt, T.M., Mahony, K. \& Pantelis, C.R. (2003). Normative data from the CANTAB. I: development of executive function over the lifespan. Journal of Clinical and Experimental Neuropsychology, 25(2), 242-254.

Mcgorry, P.D., Copolov, D.L. \& Singh, B.S. (1990). Royal park multidiagnostic instrument for psychosis: Part I. Rationale and review. Schizophrenia Bulletin, 16(3), .501-515.

Murray, R.M. \& Lewis, S.W. (1987). Is schizophrenia a neurodevelopmental disorder? British Medical Journal (Clinical Research Edition), 295(6600), 681-682.

Napal, O., Ojeda, N., Sánchez, P., Eltzagárate, E., Peña, J., Ezcurra, J. \& Gutiérrez, M. (2012). 
The course of the schizophrenia and its impact on cognition: A review of literature. Actas Espanolas de Psiquiatria, 40(4), 198-220.

Nelson, H.E. \& Willison, J.R. (1991). Restandardisation of the NART against the WAIS-R. In H. E. Nelson, ed. National Adult Reading Test (NART) Test Manual. Windsor: NFER-Nelson, 13-23.

Olivier, M.R., Killian, S., Chiliza, B., Asmal, L., Schoeman, R., Oosthuizen, P.P., Kidd, M. \& Emsley, R. (2015). Cognitive performance during the first year of treatment in firstepisode schizophrenia: a case-control study. Psychological Medicine, 45(13), 2873-83.

Owen A.M., Sahakian B.J., Semple J. Polkey, C.E., \& Robbins, T. (1995) Visuo-spatial shortterm recognition memory and learning after temporal lobe excisions, frontal lobe excisions or amygdalo-hippocampectomy in man. Neuropsychologia 33, 1-24.

Pantelis, C., Wood, S.J., Proffitt, T.M., Testa, R., Mahony, K., Brewer, W.J., Buchanan, J.A., Velakoulis, D. \& McGorry, P.D., 2009. (2009). Attentional set-shifting ability in firstepisode and established schizophrenia: Relationship to working memory. Schizophrenia Research, 112, 104-113.

Pantelis, C., Wannan, C., Bartholomeusz, C.F., Allott, K. \& McGorry, P.D. (2015). Cognitive intervention in early psychosis - preserving abilities versus remediating deficits. Current Opinion in Behavioral Sciences, 4, 63-72.

Pantelis, C., Yucel, M., Bora, E., Fornito, A., Testa, R., Brewer, W.J., Velakoulis, D. \& Wood, S.J. (2009). Neurobiological markers of illness onset in psychosis and schizophrenia: The search for a moving target. Neuropsychology Review, 19(3), 385-398.

Pantelis, C., Yucel, M., Wood, S.J., Velakoulis, D., Sun, D., Berger, G., Stuart, G.W., Yung, A., Phillips, L. \& McGorry, P.D. (2005). Structural brain imaging evidence for multiple pathological processes at different stages of brain development in schizophrenia. Schizophrenia Bulletin, 31(3), 672-696.

Prouteau, A., Verdoux, H., Briand, C., Lesage, A., Lalonde, P., Nicole, L., Reinharz, D. \& Stip, E. (2005). Cognitive predictors of psychosocial functioning outcome in schizophrenia: a follow-up study of subjects participating in a rehabilitation program. Schizophrenia Research, 77, 343-353.

Radua, J., Borgwardt, S., Crescini, A., Mataix-Cols, D., Meyer-Lindenberg, A., McGuire, P.K. \& Fusar-Poli, P. (2012). Multimodal meta-analysis of structural and functional brain changes in first episode psychosis and the effects of antipsychotic medication. Neuroscience and Biobehavioral Reviews, 36, 2325-2333.

Rodríguez-Sánchez, J.M., Ayesa-Arriola, R., Pérez-Iglesias, R., Periañez, J.A., MartinezGarcia, O., Gomez-Ruiz, E., Tabares-Seisdedos, R. \& Crespo-Facorro, B. (2013). Course of cognitive deficits in first episode of non-affective psychosis: A 3-year follow-up study. Schizophrenia Research, 150, 121-128.

de Rover, M., Pironti, V.A., McCabe, J.A., Acosta-Cabronero, J., Arana, F.S., Morein-Zamir, S., Hodges, J.R., Robbins, T.W., Fletcher, P.C., Nestor, P.J. \& Sahakian, B.J. (2011). Hippocampal dysfunction in patients with mild cognitive impairment: A functional 
neuroimaging study of a visuospatial paired associates learning task. Neuropsychologia, 49, 2060-2070.

Sigurdsson, T. \& Duvarci, S. (2015). Hippocampal-Prefrontal Interactions in Cognition, Behavior and Psychiatric Disease. Frontiers in systems neuroscience, 9(January), 190.

Stip, E., Sepehry, A.A., Prouteau, A., Briand, C., Nicole, L., Lalonde, P. \& Lesage, A. (2005). Cognitive discernible factors between schizophrenia and schizoaffective disorder. Brain and Cognition, 59, 292-295.

Thaler, N.S., Goldstein, G., Pettegrew, J.W., Luther, J.F., Reynolds, C.R. \& Allen, D.N. (2013). Developmental aspects of working and associative memory. Archives of Clinical Neuropsychology, 28, 348-355.

Toulopoulou, T., Rabe-Hesketh, S., King, H., Murray, R.M. \& Morris, R.G. (2003). Episodic memory in schizophrenic patients and their relatives. Schizophrenia Research, 63, 261271.

Vita, A., De Peri, L., Deste, G. \& Sacchetti, E. (2012). Progressive loss of cortical gray matter in schizophrenia: a meta-analysis and meta-regression of longitudinal MRI studies. Translational Psychiatry, 2, e190.

Wechsler, D. (1981). Wechsler Adult Intelligence Scale-Revised Manual, New York, NY: The Psychological Corporation.

Wechsler, D. (1987). Wechsler Memory Scale - Revised, New York, NY: Psychological Corporation.

Weinberger, D.R. (1987). Implications of normal brain development for the pathogenesis of schizophrenia. Archives of General Psychiatry, 44(7), 660-669.

Williams, L.E., Avery, S.N., Woolard, A.A. \& Heckers, S., 2012. (2012). Intact relational memory and normal hippocampal structure in the early stage of psychosis. Biological Psychiatry, 71, 105-113.

Wood, S.J., Tarnawski, A.U., Proffitt, T.M., Brewer, W.J., Savage, G.R., Anderson, V., McGorry, P.D., Velakoulis, D. \& Pantelis, C. (2007). Fractionation of verbal memory impairment in schizophrenia and schizophreniform psychosis. Australian and New Zealand Journal of Psychiatry, 41, 732-739.

Wood, S.J., Proffitt, T., Mahony, K., Smith, D.J., Buchanan, J.A., Brewer, W., Stuart, G.W., Velakoulis, D., McGorry, P.D. \& Pantelis, C. (2002). Visuospatial memory and learning in first-episode schizophreniform psychosis and established schizophrenia: a functional correlate of hippocampal pathology? Psychological Medicine, 32, 429-38. 